\title{
An investigation of hybridization and the orbital models of molecular electronic structure for $\mathrm{CH}_{4}, \mathbf{N H}_{3}$ and $\mathrm{H}_{2} \mathrm{O}$.
}

\begin{tabular}{|r|l|}
\hline Journal: & Canadian Journal of Chemistry \\
\hline Manuscript ID & Cjc-2017-0450.R1 \\
\hline Manuscript Type: & Article \\
\hline Date Submitted by the Author: & 07-Sep-2017 \\
\hline Complete List of Authors: & $\begin{array}{l}\text { Brion, C.; University of British Columbia, Chemistry } \\
\text { Wolfe, Saul; Simon Fraser University } \\
\text { Shi, Zheng; Simon Fraser University, Chemistry } \\
\text { Cooper, Glyn; University of British Columbia, Chemistry } \\
\text { Zheng, Yenyou Jeff; University of British Columbia, Chemistry }\end{array}$ \\
\hline $\begin{array}{r}\text { Is the invited manuscript for } \\
\text { consideration in a Special } \\
\text { Issue?: }\end{array}$ & N/A \\
\hline Keyword: & $\begin{array}{l}\text { hybrid orbitals, localized and delocalized orbitals, calculated and } \\
\text { experimental valence electron probability distributions }\end{array}$ \\
\hline \multicolumn{2}{|c}{} \\
\hline
\end{tabular}




\section{An investigation of hybridization and the orbital models of molecular electronic structure for $\mathrm{CH}_{4}, \mathrm{NH}_{3}$ and $\mathrm{H}_{2} \mathrm{O}$.}

C. E. Brion, Saul Wolfe, Zheng Shi, Glyn Cooper and Yenyou Jeff Zheng

C.E. Brion, G. Cooper and Y.J. Zheng. Department of Chemistry, University of British Columbia, Vancouver, BC V6T 1W5, Canada

S. Wolfe and Z. Shi. Department of Chemistry, Simon Fraser University, Burnaby, BC V5A 1S6, Canada

Corresponding author: C.E. Brion (email: brion@chem.ubc.ca) 
Abstract: Observations of the physical behaviour (motions) of the valence (frontier) electrons in the iconic benchmark hydride molecules $\mathrm{CH}_{4}, \mathrm{NH}_{3}$ and $\mathrm{H}_{2} \mathrm{O}$ are used to provide a direct experimental evaluation of hybridization, and of the localized and delocalized orbital models of molecular electronic structure, after more than 80 years since the initial proposals of these theoretical concepts. Our previously published experimental measurements of valence (frontier) electron momentum probability distributions, made by Electron Momentum Spectroscopy (EMS), are compared with those calculated using the localized molecular orbital (LMO) model and a range of hybrid orbital models, and also with the delocalized Canonical Molecular Orbital (CMO) and Density Functional Theory Kohn-Sham Orbital (KSO) models. In all cases, the electron momentum probability distributions calculated with LMOs and localized hybrid orbital type models are inconsistent with the experimental observations. In contrast those calculated with the delocalized CMOs and KSOs are in very good agreement with the experimental measurements. These findings are of importance in those research applications such as reactivity, drug and novel material design, and also in molecular electronics, where the shapes and orientations of particular valence (frontier) orbital electron density probability distributions (and not the total electron densities) are considered to be key determining factors. These findings and their implications are also of pedagogical significance in chemistry and molecular physics.

Key words: hybrid orbitals, localized and delocalized orbitals, calculated and experimental valence electron probability distributions 
Graphic abstract

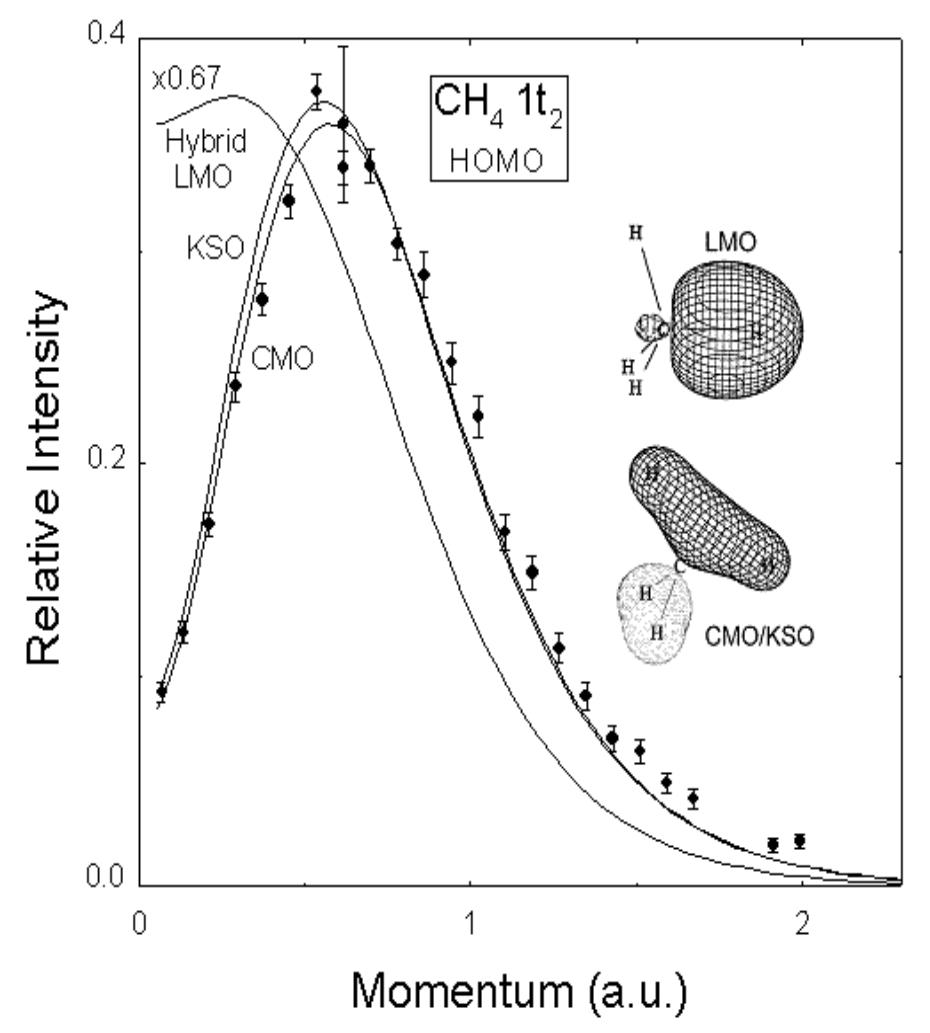




\section{Introduction}

Adequate visual and theoretical orbital models of molecular electronic structure are essential for obtaining a better understanding of fundamental phenomena in research applications such as molecular design, predictions of chemical reactivity and molecular electronics as well as in the teaching of chemistry and physics. The efficacy of these orbital models and associated theories would best be assessed by comparison with direct experimental measurements of the actual physical behavior (i.e. the position and/or momentum electron probability distributions) of the valence electrons in molecules. Many decades after the various orbital theoretical models were initially proposed, such "orbital imaging" experiments have become possible in momentum space $^{1}$ using Electron Momentum Spectroscopy (EMS). We have earlier compared EMS experimental observations of the valence electrons of methanol $\left(\mathrm{CH}_{3} \mathrm{OH}\right){ }^{2}$ the aromatic compound pyridine $\left(\mathrm{C}_{5} \mathrm{H}_{5} \mathrm{~N}\right){ }^{3}$ and also the much larger tricyclic molecule ${ }^{4} \quad 1,4$ diazabicyclo[2.2.2] Joctane $\mathrm{N}_{2}\left(\mathrm{C}_{2} \mathrm{H}_{4}\right)_{3}$ (also known as $\mathrm{DABCO}$ ), with hybridized orbitals and the localized and delocalized orbital models. For each of these larger molecular systems the measured EMS valence orbital electron momentum probability distributions have been found to be in good agreement with delocalized orbital models, but they are inconsistent with calculations using hybridized and localized orbital model calculations.

It is of fundamental interest and practical importance to see whether these findings $\mathrm{s}^{2-4}$ for this subset of larger molecules are pervasive in the molecular world by extending these investigations to the benchmark, textbook molecules $\mathrm{CH}_{4}, \mathrm{NH}_{3}$ and $\mathrm{H}_{2} \mathrm{O}$. In the present work previously published high momentum resolution EMS measurements ${ }^{1,5-7}$ for the valence electrons of these three iconic second row hydride molecules are compared with calculations using a range of hybridization models and with localized and delocalized orbital model calculations. In these previous EMS studies of the second row hydrides ${ }^{1,5-7}$ only delocalized 
orbital calculations using the canonical molecular orbital (CMO) and Density Functional Theory Kohn-Sham orbital (KSO) models were compared with experiment, but hybridization and localized orbital model calculations were not considered.

\section{Background}

A 1960 American Chemical Society award lecture ${ }^{8}$ by Robert Mulliken was entitled "What are the Electrons Really Doing in Molecules?" Correct answers to this question are key to understanding chemical and physical behavior at a fundamental electronic level. Chemical reactivity and bonding have long been considered to be determined predominantly by the behavior of the valence (outermost, or frontier) orbital electrons in atoms and molecules. The most useful orbital models will be those that are physically sensible, i.e. those that describe well the observed actual physical behavior of the valence electrons. The better the description of the observed behavior in a wide range of molecules the more powerful a model will be for interpreting natural phenomena, for example in the design of novel materials, in molecular electronics, and for predicting chemical and physical behavior in various situations. It is important to note that computational methods that are based on models that are not physically sensible can sometimes give numerically correct results in limited situations, e.g. Bohr theory calculations of the electronic spectral lines (energy levels) and ionization energies of the $\mathrm{H}$ atom and all other one electron species. However, as is well known the Bohr theory (1913) fails for all many-electron species because electrons do not physically travel in the planar circular electron orbits which were a fundamental but erroneous assumption in this elegant and ground-breaking theory which, despite its failings, introduced the important concept of quantization of energy levels in atoms. 
Soon after the landmark quantum wave mechanical description of the hydrogen atom ${ }^{9}$ by Schrodinger in 1926 electronic structure theory was extended to molecules, using the orbital approximation. This resulted in two fundamentally different orbital theories of molecular electronic structure and bonding first reported by Linus Pauling ${ }^{10}$ in 1931, and by Robert Mulliken ${ }^{11,12}$ in 1932, respectively. Following the rigorous valence bond quantum mechanical treatment of the $\mathrm{H}_{2}$ molecule in 1927 by Heitler and London, ${ }^{13}$ Pauling proposed ${ }^{10}$ a simple qualitative description of molecular electronic structure and chemical bonding in polyatomic molecules using orbitals localized on the individual component atoms. For this Pauling ${ }^{10}$ assumed the validity of the, by then widely accepted, empirical, pre-quantum, static (localized electron pair), classical ideas of G. N. Lewis. ${ }^{14}$ However in extending this 'atoms in molecules' approach from diatomic to polyatomic molecules such as methane $\left(\mathrm{CH}_{4}\right)$ it was necessary for Pauling to devise ${ }^{10}$ the additional concept of hybridization (i.e. localized hybrid atomic orbitals) and thus of localized molecular orbitals (LMOs) in order to rationalize the geometric structure and bonding. At about the same time Mulliken ${ }^{11,12}$ presented an alternative quantum mechanically based model involving delocalized canonical molecular orbitals (CMOs) in which the valence electrons in the outermost shell of a molecule generally 'orbit' the entire structure rather than the individual atoms in the molecule.

In 1965 Walter Kohn and Lu Sham ${ }^{15}$ proposed a further refined type of delocalized orbital model, namely Kohn-Sham Density Functional Theory (KS-DFT) which has since developed into a powerful method in modern computational quantum chemistry and physics. They described KS-DFT as "the Hartree-Fock (i.e.CMO) method corrected for correlation". ${ }^{15}$ Electron correlation is introduced via the exchange-correlation potential and this DFT method invokes delocalized Kohn-Sham Orbitals (KSOs). In the delocalized CMO and KSO models there are no explicit, localized, chemical bonds as such but rather a bound system of electrons and nuclei. 
Almost immediately a controversy arose in the 1930's as to the validity and relative merits of these different orbital and bonding theories for molecules, but unfortunately, until the advent of EMS many decades later, no direct experimental method was available that was capable of testing the efficacy of these very different orbital models. ${ }^{10-12,15}$ As a result this debate has continued for more than 80 years right up to the present. However even at an early stage there was very serious questioning of Pauling's ideas and methodology from many prominent and well respected scientists involved in the early development of quantum mechanics and molecular electronic structure. This included the opinions not only of Mulliken ${ }^{11,12,16,17}$ but also those of Heisenberg, ${ }^{18,19}$ Van Vleck and Sherman, ${ }^{20}$ and even apparently those of both Heitler ${ }^{21-}$ ${ }^{23}$ and London. ${ }^{24-25}$

Further illuminating and perceptive critiques of Pauling's ideas were voiced by the eminent British theoretical chemist Charles Coulson over an extended period from 1952 to $1974 .^{26-31}$ Coulson was concerned not only about the concept of hybridization but also increasingly about the validity of the idea of the localized electron pair bond. Coulson ${ }^{26}$ argued in the preface of the first edition of his book "Valence" that "chemists should not be happy with an electronic theory of valence couched in pre-wave mechanical terms". According to Simoes and Gavroglu ${ }^{21}$ Coulson was also "rather uneasy that none of these concepts (i.e. hybridization, localization etc) could be linked to a directly measurable quantity". Later Coulson ${ }^{26}$ in the second edition of 'Valence' stated: "We must not, however, allow ourselves to believe that it (i.e. hybridization) represents any real phenomenon". In 1955 Coulson $^{29}$ further said that in his opinion the chemical bond was "a convenient fiction" and many years later in $1973^{31}$ he was still referring to it as "a figment of our imagination".

Not withstanding the many serious concerns, reservations and criticisms of his theory, both early on, and later, Pauling continued to vigorously promote the localized orbital electron 
description and the concept of hybrid orbitals, ${ }^{10}$ for example in the case of $\mathrm{CH}_{4},{ }^{32,33}$ right up to the end of his long life and fruitful career. As a result his ideas involving hybrid orbitals and localized orbitals have continued in widespread use in both research applications, ${ }^{34,35}$ as well as in teaching, ${ }^{36}$ in both chemistry and molecular physics. Pauling's qualitative pictorial ideas of hybridization and localized valence bond orbital concepts initially had the advantage of providing simple pictures of the orbitals which could readily be used in discussing bonding and reaction schemes. These simple pictures also appealed to many chemists because of the minimal mathematics involved and they seemed to fit in, and provide continuity with, the conventional ideas of the localized electron pair bond and the pre-quantum, classical ideas of Lewis to which many chemists had become accustomed. In contrast, Mulliken did not include CMO pictures in his early publications and this inhibited the acceptance and use of MO theory by much of the chemical community for quite a while.

As is well-known, TOTAL wavefunctions (or TOTAL electron probability densities) from quantum mechanical theory using any complete orbital basis set permit the calculation of properties of the TOTAL system (molecule) such as total energy, molecular geometry, dipole moment etc. However, in contrast, it is not always appreciated that for predictions of important phenomena such as chemical reactivity, ${ }^{37,38}$ novel material and drug design ${ }^{39}$ and molecular electronics $^{40-43}$ in chemistry and molecular physics, ${ }^{44}$ a detailed knowledge of particular VALENCE ORBITAL electron density probability distributions is required. Hoffmann has referred to this important distinction in a recent review article ${ }^{45}$ in which he says: "The really interesting question to me is, what kind of observable is reactivity? Does it depend on one orbital (frontier control) or many?" Since the various types of valence orbital division (e.g., localized/hybridized orbitals, or delocalized CMOs/KSOs) in general exhibit spatially very different shapes and directions of the respective orbital electron density probability distributions (see figures in Results section below), then the choice of orbital model, will clearly be of critical 
importance. The situation must be that the localized/hybridized model and the delocalized orbital model cannot both be correct (i.e. physically sensible), i.e. one of them is essentially correct, or both are incorrect. However it is important to re-emphasize that for calculating properties of the TOTAL system, any complete set of orbitals (unitary transform) will give the same result - regardless of whether the particular types of orbital/pieces have any physical significance or not (i.e. you can slice a pizza any way you wish and the pieces still add up to the whole!). In this regard it should be noted again that LMOs and hybrid orbitals were simply chosen initially by Pauling, ${ }^{10}$ and later on implicitly by others, so as to correspond to the supposed localized electron pair chemical bonds as defined in the classical, pre-quantum G. N. Lewis, purely empirical, localized view of the behavior of electrons, and to the perceived bonds and observed nuclear geometry in molecules. In contrast CMOs result directly from the Hartree-Fock procedures without any such additional assumptions. The complete set of CMOs can be converted into a set of LMOs, and vice-versa, or into any other set of component pieces, by a unitary transform. Complete sets of LMOs are typically obtained from a complete set of delocalized CMOs in this manner and empirically selected to give a set of localized orbitals conforming to the classical 'ball and stick' localized chemical electron pair bond structure. ${ }^{46}$ Several such localization procedures have been reported including those by Foster and Boys ${ }^{47}$ and also by Edmiston and Ruedenberg. ${ }^{48}$

\section{Calculations and experimental details}

As shown in our earlier paper and references therein ${ }^{1}$ the "orbital" electron momentum distributions measured by EMS can be usefully compared with Target Hartree-Fock and Target Kohn-Sham, Plane Wave Impulse Approximation (PWIA) calculations, using Fourier transformed position space wavefunctions of varying levels (basis sets), and orbital models 
such as delocalized CMOs or the delocalized KSOs of Density Functional Theory (DFT). However in our earlier work on $\mathrm{CH}_{4}, \mathrm{NH}_{3}$ and $\mathrm{H}_{2} \mathrm{O},{ }^{1,5-7}$ no comparisons were made with calculations of localized and hybridized orbital models.

In the present work we extend our earlier studies to include comparison of the previously obtained EMS experimental results ${ }^{1,5-7}$ for the outer valence electrons of $\mathrm{CH}_{4}, \mathrm{NH}_{3}$ and $\mathrm{H}_{2} \mathrm{O}$ with calculations of the electron momentum probability distributions for LMOs, hybrid orbitals and hybrid orbital-atomic orbital overlaps, as well as with delocalized CMOs and KSOs. The B3LYP DFT gradient corrected functional ${ }^{49}$ was used for the KSO calculations. All calculations of the various electron momentum probability distributions were done using the GAUSSIAN 98 program $^{50}$ and the same diffuse polarized $6-311++G^{* *}$ basis set ${ }^{51}$ so as to compare the different orbital models at the same computational level. Further details on the computational procedures can be found in our earlier publications. ${ }^{1,2-4}$ The LMOs and hybrid orbitals were calculated using the Boys localization procedures ${ }^{47}$ of the MONSTERGAUSS ${ }^{52}$ program which, in the version available to us, does not handle basis sets larger than $6-311++G^{* *}$. As shown earlier ${ }^{53}$ for $\mathrm{NH}_{3}$ and other larger nitrogen containing molecules this basis set provides descriptions of delocalized KSOs and CMOs quantitatively similar to those obtained with much larger basis sets. No previous $6-311++\mathrm{G}^{* *}$ calculations for $\mathrm{CH}_{4}$ and $\mathrm{H}_{2} \mathrm{O}$ have been reported. Use of different localization procedures is not expected to lead to significantly different results because it is well known that the different methods ${ }^{54}$ for localizing orbitals give essentially the same results. The MOMAP and RESFOLD programs developed at UBC ${ }^{1}$ were used to generate the various calculated electron momentum probability distributions and 3-D position space orbital pictures.

We have previously reported the high momentum resolution EMS experimental measurements for $\mathrm{CH}_{4}, \mathrm{NH}_{3}$ and $\mathrm{H}_{2} \mathrm{O}^{1,5-7}$ used in the present evaluation of hybridization and the different orbital 
models. EMS is a high energy electron impact ionization, coincidence experiment which has the well-demonstrated capability of measuring the electron momentum probability distributions of selected valence ("orbital") electrons in atoms and molecules. ${ }^{1,55,56}$ Details of the EMS method and underlying collision theory are explained and referenced in our earlier precursor publication. ${ }^{1}$ Coincidence events are collected using repeated scans and signal averaging until acceptable statistics are obtained (approximately one to two weeks per orbital for the measurements shown in the present work).

It is important to note, as stressed in our earlier paper, ${ }^{1}$ that under the carefully chosen kinematic conditions of the EMS method, the underlying binary encounter collision theory indicates that the experiment effectively measures the momentum of the struck (ionized) electron immediately prior to "knock out". Thus it is an initial state property (i.e. a property of the electron in the neutral molecule, and not a property of the ion) that is being measured (see our earlier paper $^{1}$ and references therein for a more detailed discussion of the underlying and well-understood collision physics). The fact that the EMS measurement of the electron momentum distribution is probing an initial state property is ably demonstrated by the elegant EMS experiments of Lohmann and Weigold ${ }^{57}$ who measured the electron momentum probability distribution in the ground state of atomic hydrogen. The result of this landmark measurement is in excellent agreement ${ }^{57}$ with the (exact) solution of the Schrodinger equation ${ }^{9}$ for the ground state of atomic hydrogen. Our earlier published experimental and computational study of $\mathrm{CH}_{3} \mathrm{OH}^{2}$ compared the measurements with the theoretical electron momentum distributions calculated according to EMS theory at the most fundamental level, i.e. the absolute square of the Fourier transform of the direct full overlap between the initial neutral $((\mathrm{N})$-electron) and final ion $\left((\mathrm{N}-1)\right.$-electron) target wavefunctions. ${ }^{55}$ For this investigation we used very high level MRSD-Cl wavefunctions for both the neutral target and the final state ion. The resulting Dyson orbital overlaps are in excellent agreement not only with experiment but also with the respective 
initial state CMOs and KSOs calculated using the Target Hartree-Fock and Target Kohn Sham approximations, but they are very different from the LMO and hybrid orbital calculations. ${ }^{2}$ Similarly our previous studies of $\mathrm{CH}_{4}, \mathrm{NH}_{3}$ and $\mathrm{H}_{2} \mathrm{O}^{1,5-7}$ show that the calculated MRSD-Cl Dyson orbitals are in good agreement with the EMS experiments and with the respective CMOs and KSOs for the target molecules. These findings ${ }^{1,2,5-7}$ further confirm that EMS measurements essentially probe the initial state (i.e. the neutral molecule) and that the orbital and Dyson orbital calculations give essentially the same momentum distributions for delocalized electrons. Thus it can be expected that Dyson orbital overlap calculations in the localized description would lead to a momentum distribution essentially the same as that for the target LMO corresponding to the removed electron.

EMS is a momentum space experiment, and the momentum space representation, both in experiment and computation, although familiar to physicists, is not always well understood by chemists who are generally much more conversant with position space concepts of electron probability density distributions and wavefunctions. A position $(r)$ space wavefunction $\Psi(r)$ is related to its momentum $(p)$ space counterpart $\Psi(p)$ by a Fourier Transform (FT). It is possible to "do" quantum mechanics in momentum (i.e. reciprocal) space. In the EMS experiment, the momentum probability distribution of a given molecular electron is determined (i.e. the momentum probability distribution $|\Psi(p)|^{2}$ rather than the position probability distribution $|\Psi(r)|^{2}$ of the electron in the molecule is measured). Elsewhere in modern science, FT methods using measurements in reciprocal space have enabled difficult, or seemingly impossible, types of measurement to be made. For example no widely applicable method of directly observing position space electron densities of selected valence electrons has, thus far, been devised. However momentum space imaging became possible with the advent of EMS and as such it is a Fourier Transform (FT) technique. Scientists today are familiar with FT techniques such as 
FTNMR which is also used in MRI medical imaging. Another example is X-ray crystallography which enabled the determination of crystal lattice spacings by Fourier Transformation of diffraction ( $k$ space) patterns since no method for direct position space measurement of such quantities was available.

\section{Results}

By 1935 Mulliken had already discussed the $\mathrm{MO}$ electronic structures of $\mathrm{CH}_{4},{ }^{58}$ and also of $\mathrm{NH}_{3}$ and $\mathrm{H}_{2} \mathrm{O} .^{59}$ Nearly 40 years ago low momentum resolution studies of $\mathrm{CH}_{4}{ }^{60}$ at The Flinders University of South Australia, and of $\mathrm{NH}_{3}{ }^{61}$ and $\mathrm{H}_{2} \mathrm{O}^{62}$ at The University of British Columbia, were among the very first EMS experimental measurements to be reported for molecules. However in these initial studies ${ }^{60-62}$ only primitive delocalized orbital wavefunctions were used in the calculations, and no comparisons were made with hybrid and localized orbital models. Our later high momentum resolution EMS measurements for $\mathrm{CH}_{4}, \mathrm{NH}_{3}$ and $\mathrm{H}_{2} \mathrm{O}^{1,5-7}$ used in the present study, combined with the presently reported calculations of the orbital electron momentum probability distributions, should now permit a definitive assessment as to the physical significance, or otherwise, of hybridization and the different orbital models. This should finally provide unambiguous clarification of the long-standing uncertainty which has been the focus of so much debate for the past 80 years. Let it again be stressed that our experiments measure an actual physical property (i.e. the electron momentum probability distribution) of the valence (frontier) electrons in the respective orbitals of the neutral target molecules. In science, reliable direct experiment is the "Acid Test" for theory and modeling. A recent new multichannel EMS study by Liu Yuan et $\mathrm{al}^{63}$ confirms our earlier high momentum resolution experimental measurements ${ }^{1,5-7}$ of the valence electron momentum probability distributions of $\mathrm{CH}_{4}, \mathrm{NH}_{3}$ and $\mathrm{H}_{2} \mathrm{O}$. 
The present calculations for hybridization and the three different (LMO, CMO and KSO) orbital models are shown below compared with our previously published experimental measurements ${ }^{1,5-7}$ of the valence electron momentum probability distributions for $\mathrm{CH}_{4}, \mathrm{NH}_{3}$ and $\mathrm{H}_{2} \mathrm{O}$. In each case three-dimensional pictures of the respective more familiar position space orbitals, calculated with the same basis sets as the electron momentum distributions, are also shown. The LMO/hybrid orbitals were obtained using the Foster and Boys localization procedures $^{47}$ and they correspond to the least tightly bound electrons in the simple valence bond theory quadra-degenerate bond pair electrons for $\mathrm{CH}_{4}$, and the respective electron lone pairs in the cases of $\mathrm{NH}_{3}$ and $\mathrm{H}_{2} \mathrm{O}$. In the case of $\mathrm{CH}_{4}$ all four degenerate $\mathrm{LMO}$ /hybrid orbitals will lead to the same momentum distribution due to the conditions of the EMS experiment for the randomly oriented target molecules. ${ }^{55}$

Figure 1 shows the CMO, KSO and LMO (effectively an $\mathrm{sp}^{3}$ hybrid/H 1 s overlap) momentum probability distribution calculations for the valence shell electrons in the methane molecule $\left(\mathrm{CH}_{4}\right)$ together with our earlier reported EMS measurements. ${ }^{1,5}$ The momentum space integrated areas under the $1 t_{2}$ and $2 a_{1}$ electron momentum distributions reflect the $3: 1$ ratio expected from the state degeneracies in the delocalized molecular orbital and Kohn-Sham orbital models. $^{1,5}$ The localized hybrid/LMO calculations are clearly inconsistent with the measured electron momentum probability distributions whereas there is very good quantitative agreement with the delocalized $\mathrm{CMO}$ and $\mathrm{KSO}$ calculations. 


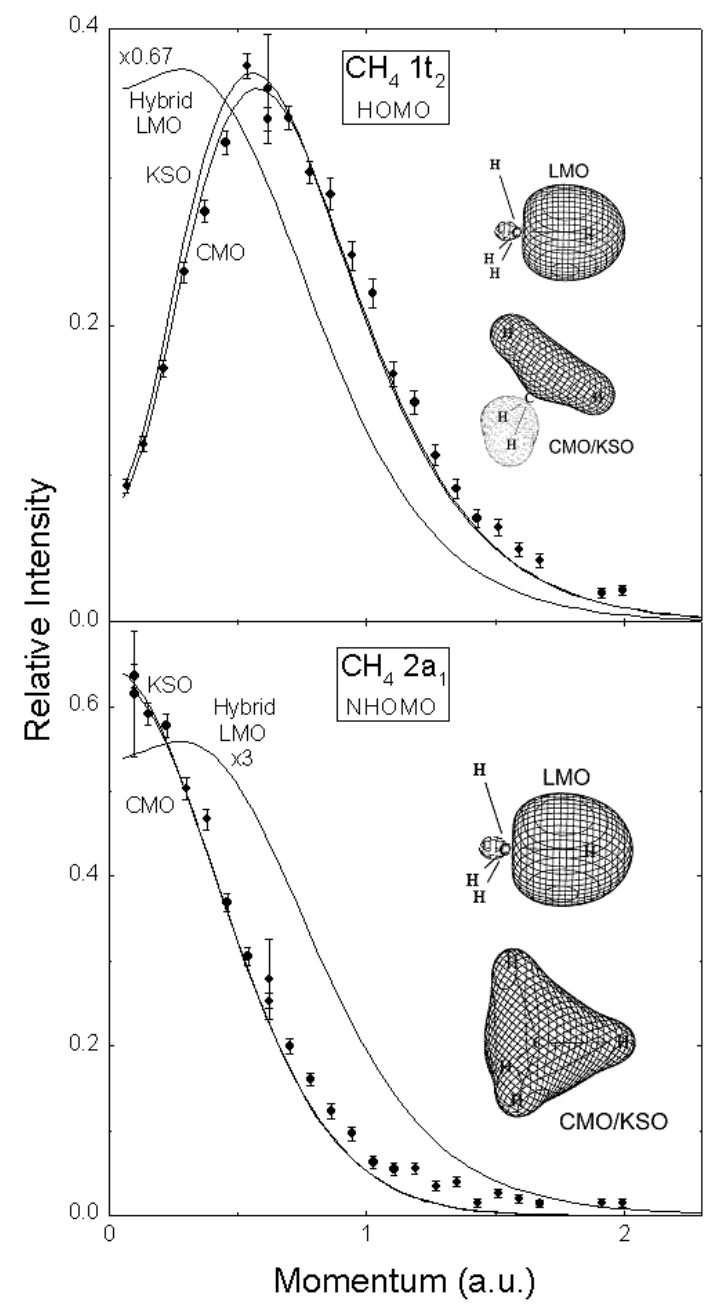

Fig. 1. Calculations and EMS measurements of the electron momentum distributions for the valence electrons of $\mathrm{CH}_{4}$ - see text for further details.

Figure 2 shows the calculated electron momentum probability distributions of $\mathrm{CH}_{4}$ for (a) the overlap of a hydrogen $1 \mathrm{~s}$ and a carbon $\mathrm{sp}^{3}$ hybrid orbital $\left(\mathrm{sp}^{3} \mathrm{~s}\right)$, (b) the LMO, (c) the hybrid orbital on carbon $\left(s p^{3}\right)$, and $(d)$ the delocalized $1 t_{2} \mathrm{CMO} / \mathrm{KSO}$. It can be seen that only the $1 \mathrm{t}_{2}$ CMO/KSO delocalized calculations have the experimentally observed "p-type" shape momentum probability distribution shown in Figure 1. All three localized hybrid/LMO possibilities predict an (incorrect) "s-type" momentum probability distribution. 


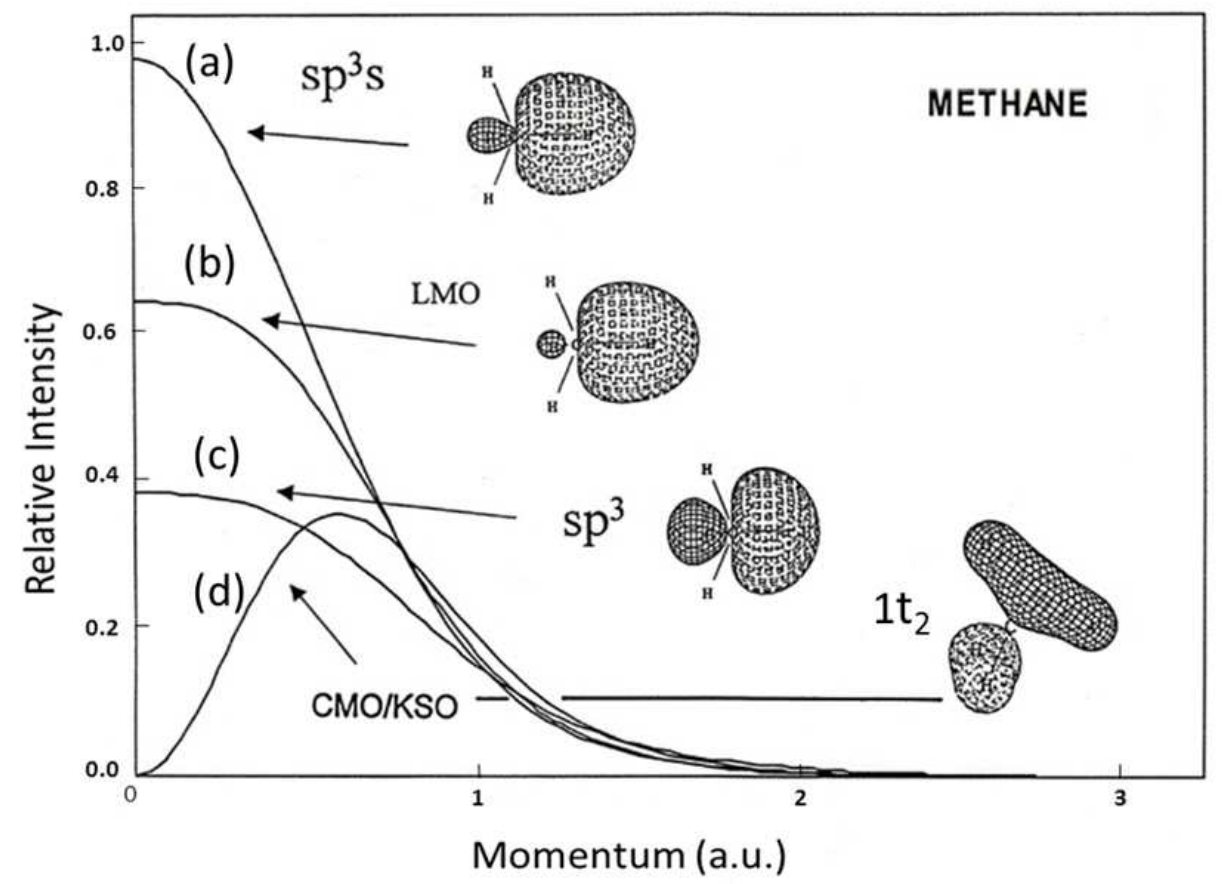

Fig. 2. Calculated electron momentum distributions for methane using localized and delocalized orbital models - see text for further details.

Figure 3 shows our earlier reported experimental EMS measurements ${ }^{1,5-7}$ and the various presently calculated electron momentum probability distributions for the outermost valence electrons of $\mathrm{H}_{2} \mathrm{O}\left(\mathrm{HOMO}\right.$ and $\mathrm{NHOMO}, \mathrm{NH}_{3}(\mathrm{HOMO})$, and also the $\mathrm{HOMO}$ of $\mathrm{CH}_{4}$ (see Figure 1) for comparison purposes. HOMO is the highest occupied molecular orbital, and $\mathrm{NHOMO}$ is the next highest occupied molecular orbital. In every case the localized hybrid/LMO calculations are clearly inconsistent with the corresponding measured electron momentum probability distributions whereas there is good and excellent agreement respectively with the CMO and KSO calculations. 

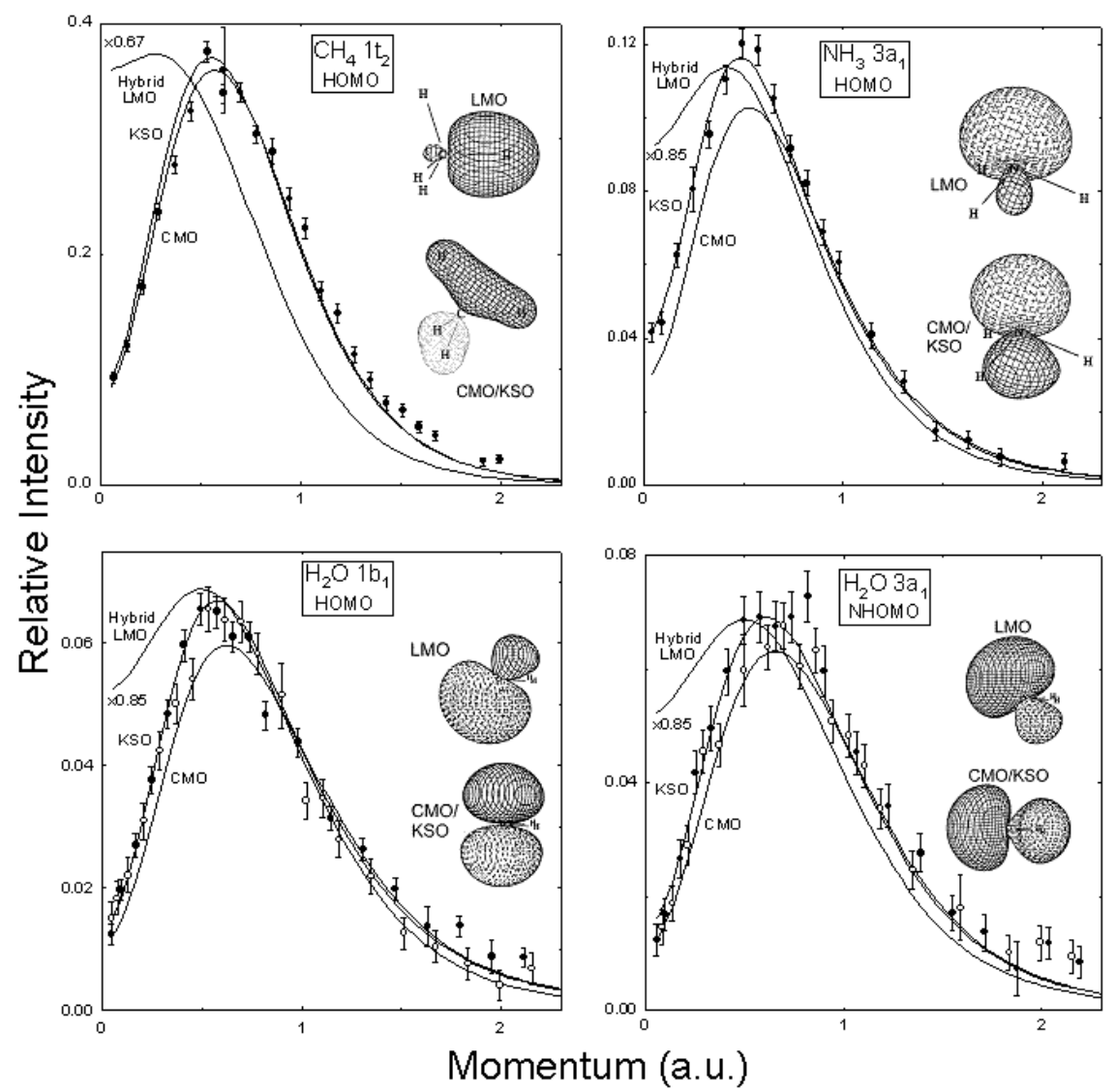

Fig. 3. Calculations and EMS measurements of the electron momentum distributions for the outer valence electrons of $\mathrm{CH}_{4}, \mathrm{NH}_{3}$ and $\mathrm{H}_{2} \mathrm{O}$ - see text for further details.

\section{Discussion}

The experimental results and calculations shown in Figures 1, 2 and 3 clearly demonstrate, directly and definitively that for each of the valence orbitals of the iconic "benchmark" row 2 hydrides, $\mathrm{CH}_{4}, \mathrm{NH}_{3}$ and $\mathrm{H}_{2} \mathrm{O}$, the delocalized $\mathrm{KSO}$ orbital model, which includes an accounting for electron correlation, reproduces the observed experimental valence electron momentum probability distributions almost exactly. The delocalized CMOs provide very good semiquantitative, or better, agreement with experiment. Conversely all of the localized/hybrid orbital model descriptions fail to reproduce the observed physical behavior of the valence electrons in 
$\mathrm{CH}_{4}, \mathrm{NH}_{3}$ and $\mathrm{H}_{2} \mathrm{O}$. This would seem to be a general situation since identical conclusions have resulted from our three earlier published comparisons of EMS measurements with LMO/hybrid, $\mathrm{CMO}$ and KSO computational studies for methanol $\left(\mathrm{CH}_{3} \mathrm{OH}\right),{ }^{2}$ for the aromatic compound pyridine $\left(\mathrm{C}_{5} \mathrm{H}_{5} \mathrm{~N}\right){ }^{3}$ and for the much larger tricyclic molecule 1,4-diazabicyclo[2.2.2]octane, $\mathrm{N}_{2}\left(\mathrm{C}_{2} \mathrm{H}_{4}\right)_{3}$ (also known as DABCO $)^{4}$ respectively. Clearly all localized and hybrid orbital model descriptions of electron behavior fail to describe the observed valence electron momentum probability distributions in every case for this broad range $\mathrm{e}^{1-7}$ of small benchmark, second row hydrides and larger polyatomic molecules. It is of importance to note that the discrepancies between the hybrid/localized orbital calculations and the EMS measurements are particularly large in the chemically sensitive ${ }^{1}$ low momentum $p$ (i.e. larger $r$ ) regions of the wavefunction (electron density). As such we may expect that Pauling's localized hybrid/LMO orbitals will be unsuitable for realistic modeling of phenomena where electron density probability distributions in frontier-type valence orbitals play a dominant determining role. In contrast, in all cases, the delocalized $\mathrm{CMO}$ and $\mathrm{KSO}$ orbital models describe the observed physical behaviour of the valence electrons very well, and these models should therefore be suitable for general use. In this regard it should further be noted that EMS studies of the valence electrons of a very large range of other organic and inorganic molecules, in our own and other laboratories, have also indicated the excellent modeling of the measurements in all cases by delocalized CMO and/or KSO descriptions. However for this very large and diverse range of other molecules, no localized/hybrid orbital calculations were performed at the time of the original studies.

The present findings for the row 2 hydrides together with the results of our earlier comprehensive studies of larger molecules ${ }^{2-4}$ strongly support the validity of Mulliken's delocalized CMO model, ${ }^{11,12}$ and also of Kohn and Sham's delocalized $\mathrm{KSO}^{15}$ orbital model in that they give a good descriptions of the observed valence orbital electron behavior in this range of different molecules. This suggests that in applications such as molecular and drug design, ${ }^{39}$ 
the prediction of reactivity ${ }^{37,38}$ and molecular electronics ${ }^{40-43}$ where the electron density probability distribution of valence electrons in given frontier orbitals is evidently an important determining factor ${ }^{45}$ it can be expected that delocalized orbital electron density calculations using high level CMOs, and especially KSOs (which include correlation), will lead to the most meaningful and reliable results. Conversely, in such applications, localized orbital descriptions such as LMOs, hybrid orbitals ${ }^{10,32,33}$ and "rabbit ears" 64,65 electron distributions, despite occasional apparent successes, are unlikely to provide consistently satisfactory results due to their different shapes and orientations compared to those of the delocalized orbital descriptions. The present experimentally based findings also affirm the earlier suggestion of Stowasser and Hoffmann ${ }^{66}$ that the KSOs of Density Functional Theory have not been accorded the reality they deserve, and also the conclusions of Baerends and Gritsenko ${ }^{67}$ who consider Kohn-Sham orbitals to be physically sound, and useful for describing chemical effects.

In $1931^{10}$ Pauling claimed that his work on hybridization: "--- has also provided a formal justification of the rules set up in 1916 by G. N. Lewis for his electron pair bond". However this seems hardly surprising since, as noted above and elsewhere, ${ }^{6,69}$ in developing his theory Pauling ${ }^{10,70}$ had assumed the validity of the basic ideas of Lewis and the localized electron pair bond! Pauling also asserted ${ }^{32}$ that in his view his simple method: "---leads to the quantitative theoretical conclusion that in the normal state the methane molecule is tetrahedral and, to the degree of approximation involved, has $s p^{3}$ bond orbitals". Our present experimentally based study and calculations for $\mathrm{CH}_{4}$ do not support the claim with regard to the electronic structure (i.e. $\mathrm{sp}^{3}$ orbitals) of $\mathrm{CH}_{4}{ }^{10,32,33}$ although our calculations and findings are, of course, consistent with the geometric structure of $\mathrm{CH}_{4}$ being tetrahedral. It is important to note that the concepts of molecular geometry (i.e. nuclear structure) and electronic structure (orbitals) are two different considerations but that they are not the same and should not be used interchangeably. 
A result similar to the classical, localized $\mathrm{sp}^{3}$-hybrid/LMO description of the electronic structure of $\mathrm{CH}_{4}$ given by Pauling is also claimed from the results of the spin-coupled calculations reported by Penotti et al. ${ }^{71}$ These authors ${ }^{71}$ state that the spin-coupled wavefunction in essence provides a "simple physical picture of the behavior of correlated electrons". In marked contrast to these conclusions, ${ }^{10,33,71}$ our own earlier very high level MRSD-Cl computational study of $\mathrm{CH}_{4}{ }^{1,5}$ shows very good agreement between the presently employed EMS experiments and the delocalized CI Dyson orbital, as well as with the near Hartree-Fock limit delocalized CMO and KSO orbital model calculations. These results ${ }^{1,5}$ compared with the calculations reported in the present investigation indicate a physical picture very different from the $\mathrm{sp}^{3}$ hybrid/LMO type orbital electronic structure model for $\mathrm{CH}_{4}$. It is noteworthy that our earlier very high level calculations for $\mathrm{CH}_{4}^{1,5}$ show a significantly lower variational total energy for the MRSD-Cl wavefunction ( -40.4297 hartrees) compared with the value of -40.2655 hartrees for the spincoupled wavefunctions obtained by Penotti et al. ${ }^{71}$ It should also be noted that our correlated multi-reference singles and doubles configuration interaction (MRSD-CI) wavefunctions ${ }^{1,5}$ include very diffuse functions, which are variationally optimized, not only for total energy (which depends mainly on the short distance $r$ (high momentum $p$ ) part of the wavefunction) but also for properties dependent on the chemically important low momentum $p$ (large distance $r$ ) region of phase space to which EMS measurements are particularly sensitive. This was a key factor in developing the extremely accurate $\mathrm{Cl}$ and Hartree-Fock wavefunctions for $\mathrm{H}_{2} \mathrm{O}, \mathrm{NH}_{3}$ and $\mathrm{CH}_{4}{ }^{1,5-}$ ${ }^{7}$ and $\mathrm{CH}_{3} \mathrm{OH}^{2}$ in our collaborations with E. R. Davidson and his co-workers.

In their spin-coupled valence bond study of $\mathrm{CH}_{4}$, Penotti et $\mathrm{al}^{71}$ also make the further claim (see page 436) that "orbitals do not 'exist', they do not correspond to physical observables". Similar claims have been made by others including Ogilvie ${ }^{72}$ and Scerri. ${ }^{73}$ Our present experimental work and all previous EMS studies for the valence electrons of a wide range of inorganic and organic molecules ranging from molecular hydrogen ${ }^{74,75}$ to targets as large as the antibacterial 
agent urotropine,$^{76}$ 1,4-diazabicyclo[2.2.2] octane (DABCO $)^{4}$ and the amino-acid glycine, ${ }^{77,78}$ as well as the more recent STM studies of adsorbates, ${ }^{79}$ fast laser tomographic imaging of $\mathrm{N}_{2}{ }^{80}$ and analysis of full-angle photoemission from molecular films by Ramsay et al ${ }^{81,82}$ have all clearly shown the situation to be otherwise and that delocalized "orbital imaging" can effectively be achieved experimentally. In this regard, it is interesting to reflect on the observations of Nobel Laureate Kenichi Fukui ${ }^{37}$ who four decades ago in 1977, at about the time EMS was just beginning, and unaware of this new instrumental development, made the following prescient observation "In the present status of experimental techniques no one has been able to observe the orbital pattern experimentally. However, to date, no one can assert that this is totally impossible. Some device to combine photoelectron spectroscopy with diffraction or scattering analysis might provide a means to gather information on the orbital pattern. If we could experimentally obtain any knowledge of HOMO or LUMO patterns, chemistry would be profoundly affected". Similarly in an interview ${ }^{83}$ in 2007, Fukui's co-Laureate Roald Hoffmann opined on the subject of orbitals: "I think orbitals are real and from orbitals we can build the world of chemistry. They are the most far-reaching and unifying way to see chemical phenomena."

It is clear then from the present work that the delocalized CMOs and KSOs are orbital models that very closely correspond to what valence electrons are really doing in molecules - see reference. ${ }^{8}$ Conversely our experiments unequivocally indicate that the individual valence electron hybrid orbitals and LMOs in Pauling's localized orbital model do not correspond to physical reality. The present experimentally based findings thus resoundingly vindicate the many reservations and cautions (see Introduction) expressed earlier by Mulliken ${ }^{11,12,16,17}$ by Van Vleck and Sherman, ${ }^{20}$ by Heisenberg, ${ }^{18,19}$ by London, ${ }^{24,25}$ by Heitler ${ }^{21-23}$ and also by Coulson ${ }^{26-31}$ as to the viability of Pauling's hybridized and localized orbital model of valence electron molecular electronic structure and chemical bonding. 
In view of the present findings, and the fact that the concepts of hybridization and LMOs were devised assuming ${ }^{10}$ pre-quantum, classical ideas it is perhaps not surprising that experiment now shows that these concepts do not correspond to physical reality. The present results are also perhaps not surprising since localization procedures ${ }^{47,48,54}$ as Csizmadia $^{46}$ has notably remarked, "---- aim to convert a quantum problem to a classical problem, which is in essence, the ball and stick model". In contrast the (now shown to be physically realistic) CMOs and KSOs result directly from the Hartree-Fock procedures without any such classical assumptions. The complete set of CMOs (or KSOs) can be converted into a set of LMOs (or LKSOs), and viceversa, or into any other desired set of component pieces, by a suitably selected unitary transform (regardless of whether the separate components have any physical significance). So it is clear that our experiments show that hybridized orbitals and LMOs are, at best, abstract mathematical functions without any real physical significance with regard to individual orbital descriptions of valence electron behavior. Their origin lies in the empirical, classical, prequantum ideas to which they were aligned. ${ }^{10}$ Our findings also provide experimental support for the recently reported views of Alexander Grushow who has questioned ${ }^{84}$ the use of hybrid (localized) orbital models in the teaching and practice of much of chemistry.

However much that localization and hybridization might have appeared to work in some situations, they have had and will likely continue to have significant problems at a deeper level. In those situations where the shapes and directions of the electron density probability distributions of frontier electrons are likely to be of crucial importance in phenomena such as, for example, in Kenichi Fukui's Frontier Electron Orbital Theory of Reactivity ${ }^{37}$ and the WoodwardHoffmann rules, ${ }^{38}$ as well as in areas such as Molecular Electronics, ${ }^{40-43}$ and Novel Material and Drug Design, ${ }^{39}$ then our findings supporting the delocalized CMO and KSO orbital models are significant. In this regard, it is noteworthy that it was our (late) senior co-author, Professor Saul Wolfe's frustration with trying to use localized/hybridized orbital models as a reliable predictive 
tool in his computationally-guided drug-design laboratory at SFU that prompted the initiation of the perhaps somewhat unlikely interdisciplinary collaboration with the UBC Chemical Physics (EMS) group. Professor Wolfe indicated that he and his group were finding that delocalized orbital models were in general a much more reliable tool than localized (hybridized) orbital models in computationally assisted drug design. The result has been our present collaborative investigative work on the iconic 'textbook' row 2 hydrides $\mathrm{CH}_{4}, \mathrm{NH}_{3}$, and $\mathrm{H}_{2} \mathrm{O}$, as well as our previous, already published, papers evaluating the three different orbital models and hybridization in methanol, $\left(\mathrm{CH}_{3} \mathrm{OH}\right),{ }^{2}$ and pyridine, $\left(\mathrm{C}_{5} \mathrm{H}_{5} \mathrm{~N}\right),{ }^{3}$ as well as the much larger molecule 1,4-diazabicyclo[2.2.2] octane, $\mathrm{N}_{2}\left(\mathrm{C}_{2} \mathrm{H}_{4}\right)_{3}$, also known as $\mathrm{DABCO},{ }^{4}$ all of which have led to the same conclusions validating the delocalized $\mathrm{CMO}$ and $\mathrm{KSO}$ orbital models. Our findings suggest that use of localized/hybridized orbital models in research endeavours is likely to prove unreliable for consistent widespread application.

The present study indicates that the delocalized CMO and in particular the delocalized KSO orbital models are really very good for describing the measured electron density probability distributions of valence electrons. Conversely localized LMO/hybrid orbital descriptions are seen to be unrealistic models. Therefore since Pauling's simple model, i.e. localized hybrid orbitals and LMOs, still continues to dominate much of the language of chemistry and discussions of chemical bonding and reactivity, not only in research but also in teaching, it is important to ask whether continuing widespread uncritical use of these concepts is justified? For example, notwithstanding the strong differences of opinion, questions and serious concerns expressed over many years, and discussed above, LMOs and hybrid orbital pictures, typically for the iconic, row 2 hydrides $\mathrm{H}_{2} \mathrm{O}, \mathrm{NH}_{3}$ and $\mathrm{CH}_{4}$, are presently featured uncritically in almost every undergraduate chemistry textbook. ${ }^{36}$ In addition many students of organic chemistry continue be taught that orbital hybridization is an important quantum mechanical concept useful for understanding molecular structure and bonding. ${ }^{85-55}$ The results of our present, and previous 
comprehensive experimental and computational investigations, ${ }^{2-4}$ challenge these views. Our findings obviously give pause for thought as to how some aspects of chemistry and molecular physics should be taught in the $21^{\text {st }}$ century. Hybridization can perhaps still be taught as part of a series of an evolving hierarchy of models because we can learn much, not least from their respective shortcomings and limitations. For example in this context, as already mentioned in the Introduction, the (unphysical) Bohr theory is still often considered as part of the curriculum on electronic structure because it introduced the important idea of quantization in the development of the quantum mechanics of many electron atoms and molecules.

While geometric structure can be accurately predicted by both MO and DFT geometry optimized calculations, and also by modern valence bond computational methods (because geometry is a property of the whole molecule), it is often rightly claimed that such calculations are not really all that feasible for undergraduates. In this regard, it is often asserted that hybrid orbitals are needed to provide a simple rationalization of geometric structures. However molecular geometry, or at least the shape of the molecule, is easily taught and predicted for many molecules by valence shell electron pair repulsion theory (VSEPR) - an empirical procedure which does not invoke the use of any mathematics or any orbital model. ${ }^{89}$

\section{Conclusions}

The present investigations for the iconic "benchmark" second row hydrides, $\mathrm{CH}_{4}, \mathrm{NH}_{3}$ and $\mathrm{H}_{2} \mathrm{O}$, as well as our earlier studies of other larger molecules, ${ }^{2-4}$ show that the experimentally observed physical behaviour (i.e. the electron momentum probability distributions) of valence (frontier) electrons is well described, in every case, by the delocalized CMO model, and even better by the correlated KSO orbital model. Conversely the localized LMO and hybridized orbital models do not match the experimental measurements. These localized models are shown to be 
unphysical (i.e. they are abstract mathematical functions obtained by a unitary transform conveniently chosen to correspond ${ }^{46}$ to pre-quantum classical ideas of bonding and molecular structure). These results lend strong support to Mulliken's delocalized Molecular Orbital Theory ${ }^{11,12}$ and to the delocalized Kohn-Sham orbitals of Density Functional Theory. ${ }^{15}$ They also strongly support, and lend substance to, the long expressed serious reservations of Mulliken, Heisenberg, Coulson and others ${ }^{11,12,16-31}$ as to the viability of the concept of the localized electron pair bond and of Pauling's model ${ }^{10}$ of hybridization and localized molecular orbitals. Implications for both research and teaching have been considered.

In much of science, experiment has usually historically preceded the development of the corresponding theory needed for interpretation and prediction purposes. However this has not been the situation in the case of the orbital models of chemistry and the concept of hybridization, where no direct experimental test was, even in principle, possible until the advent of EMS more than 40 years after the development of the simple valence bond/hybridization/LMO and Molecular Orbital theories of molecular electronic structure in the late 1920s and the early 1930s. If the cards had been dealt differently in history and the results of the present EMS measurements, as well as those of the more recently developed techniques of STM imaging of adsorbates, ${ }^{79}$ Laser Tomographic Imaging $^{80}$ and photoemission imaging ${ }^{81,82}$ had preceded the development of the various orbital models in molecular quantum mechanics, theorists would undoubtedly have claimed that the delocalized CMO and KSO orbital models of valence electron behavior were really pretty good, whereas the localized/hybrid and LMO orbital models were not physically realistic. This historical order however was not the case here and this has probably resulted in much of the ongoing delocalized orbital and hybrid/localized orbital controversy, as well as the semantics as to whether orbitals do, or do not, exist. ${ }^{37,66,67,72,73,79-83}$ Further, even more serious questioning and examination of the robustness ${ }^{90}$ of the concept of the localized electron pair chemical bond would also surely have taken place at an earlier 
juncture. The present work suggests that some reassessment of the bonding and electronic structure aspects of chemistry and molecular physics is needed in the $21^{\text {st }}$ century.

\section{Acknowledgments}

We are indebted to our late co-author and friend Professor Saul Wolfe for his profound insights and for originally raising the possibility of this collaborative interdisciplinary project between the groups at UBC (EMS and Chemical Physics) and SFU (Experiments and Theoretical Modeling in Organic Chemistry and Drug Design). Helpful discussions over the years with Kenichi Fukui, Walter Kohn, Ian McCarthy, Erich Weigold, Noam Weinberg, Imre Csizmadia and Joseph Murdoch, as well as with many others, are also gratefully acknowledged. We thank NSERC Canada for Operating and Equipment Grants, and The Peter Wall Institute of Advanced Studies at UBC for a Major Thematic Grant. One of us (CEB) wishes to acknowledge the hospitality of the Atomic and Molecular Physics Group at The Australian National University where most of our foundational paper ${ }^{1}$ was written and where much of the library research and early drafting for the present related manuscript was carried out. 


\section{References}

(1) Brion, C. E.; Cooper, G.; Zheng, Y.; Litvinyuk, I. V.; McCarthy, I. E. Chem. Phys. 2001, 270,13 and references therein.

(2) Rolke, J.; Zheng, Y.; Brion, C.E.; Shi, Z.; Wolfe, S.; Davidson, E.R. Chem. Phys. 1999, $244,1$.

(3) Tixier, S.; Shapley, W. A.; Zheng, Y.; Chong, D. P.; Brion, C. E.; Shi, Z.; Wolfe, S. Chem. Phys. 2001, 270, 263.

(4) Wolfe, S.; Shi, Z.; Brion, C. E.; Rolke, J.; Zheng, Y.; Cooper, G.; Chong, D. P. Can. J. Chem. 2002, 80, 222.

(5) Clark, S. A. C; Reddish, T. J.; Brion, C. E.; Davidson, E. R.; Frey, R. Chem. Phys. 1990, $143,1$.

(6) Bawagan, A. O.; Müller-Fiedler, R.; Brion, C. E.; Davidson, E. R.; Boyle, C. Chem. Phys. $1988,120,335$.

(7) Bawagan, A. O.; Brion, C. E.; Davidson, E. R. Feller, D. Chem. Phys. 1987, 113, 19.

(8) Mulliken, R. S. The Vortex 1960, 21, 182 (ACS G. N. Lewis Award Lecture).

(9) Schrodinger, E. Ann. Phys. 1926, 79, 361.

(10) Pauling, L. J. Amer. Chem. Soc. 1931, 53, 1367.

(11) Mulliken, R. S. Phys. Rev. 1932, 40, 55.

(12) Mulliken, R. S. Phys. Rev. 1932, 41, 49.

(13) Heitler, W.; London, F. Z. Physik 1927, 44, 455.

(14) Lewis, G. N. J. Amer. Chem. Soc. 1916, 38, 762.

(15) Kohn, W.; Sham, L. J. Phys. Rev. 1965, 140, A1133.

(16) Mulliken, R. S. Chem. Rev. 1931, 9, 347.

(17) Mulliken, R. S. J. Chem. Phys. 1965, 43, S2.

(18) Simoes. A.; Gavroglu, K. Stud. Hist. Phil. Mod Phys. 2000, 31, 511. 
(19) Heisenberg, W. Chemistry at the Centenary Meeting of the British Association for the Advancement of Science (Cambridge: W. Heffer and and Sons Ltd., 1932, p.247).

(20) Van Vleck, J. H.; Sherman, A. Rev. Mod. Phys. 1935, 7, 167.

(21) Gavroglu, K.; Simoes, A. in Communicating Chemistry - Textbooks and Their Audiences, edited by A. Lundgren and B. Bensaude-Vincent (Science History Publications/USA, 2000, 1789-1939).

(22) Gavroglu, K.; Simoes, A. Neither Physics Nor Chemistry: A History of Quantum Chemistry (MIT Press, 2012).

(23) Gavroglu, K.; Simoes, A. Hist. Stud. Phys. Sci. 1994, 25, 47.

(24) Mason, S. F. Chem. Soc. Rev. 1997, 29.

(25) Gavroglu, K. "Fritz London - a scientific biography”, Cambridge University Press (2008).

(26) Coulson, C. A. in Valence, (Oxford University Press, first edition 1952, second edition 1961, third edition (R. McWeeny) 1979).

(27) Coulson, C. A. Rev. Mod. Phys. 1960, 32, 170.

(28) Coulson, C. A. Sci. J. Royal College Science, 1952, 21, 11.

(29) Coulson, C. A. J. Chem. Soc. 1955, 2069.

(30) Coulson, C. A. Pure Appl. Chem. 1970, 24, 257.

(31) Coulson, C. A. in Theoretical Chemistry - Past and Future, edited by S. L. Altmann (Clarendon Press, Oxford, 1974, p. 20).

(32) Pauling, L. J. Chem. Educ. 1992, 69, 519.

(33) Kauffmann, G. B.; Kauffmann, L. M. J. Chem. Educ. 1996, 73, 29.

(34) Grundberg, H.; Eriksson-Bajtner, J.; Bergquist, K.; Sundin, A.; Ellervik, U. J. Org. Chem. 2006, 71, 5892 .

(35) Hersh, W. H.; Lam, S. T.; Moskovic, D. J.; Panagiotakis, A.J. J. Org. Chem. 2012, 77, 4968.

(36) Zuhmdahl, S. S. Chemical Principles (Houghter-Mifflin, 1998). 
(37) Fukui, K. Int. J. Quant. Chem. 1977, 12 (Suppl.1), 277.

(38) Woodward, R. B.; Hoffmann, R. The Conservation of Orbital Symmetry (Verlag Chemie and Academic Press, Weinheim and New York, 1970).

(39) Wolfe, S.; Jin, H.; Yang, K.; Kim, C.; McEachern, E. Can. J. Chem. 1994, 72, 1051.

(40) Tsuji, Y. T.; Hoffmann, R. Angew. Chem. Int. Ed. 2014, 53, 4093.

(41) Bruot, C.; Hihath, J.; Tao, N. Nature Nanotech. 2012, 7, 35.

(42) Heath, J. R. Annu. Rev. Mater. Res. 2009, 39, 1.

(43) Petty, M. C.; Bryce, M. R.; Bloor, D. Introduction to Molecular Electronics (Oxford University Press, New York, 1995).

(44) Coulson, C. A. in Molecular Orbitals in Chemistry, Physics, and Biology' - A Tribute to R. S. Mulliken, edited by Per-Olov Lowdin and B. Pullman (Academic Press New York, 1964).

(45) Hoffmann, R.; Shaik, S.; Hiberty, R. C. Acc. Chem. Res. 2003, 36, 750, see comment by Hoffmann, R. on page 755.

(46) Csizmadia, I. (private communication).

(47) Foster, M.; Boys, S. F. Rev. Mod. Phys. 1960, 32, 296.

(48) Ruedenberg, K.; Edmiston, C. Rev. Mod. Phys. 1963, 35, 457.

(49) Stephens, R. J.; Devlin, F.; Chabalowski, C. F.; Frisch, M. J. J. Chem. Phys. 1994, 98, 11623.

(50) Frisch, M. J. et al (1998), Revision A.6., (Gaussian Inc., Pittsburgh PA 1998).

(51) Krishnan, R.; Binkley, J. S.; Seeger, R.; Pople, J. A. J. Chem. Phys. 1980, 72, 650.

(52) Peterson, M. R.; Poirier, R. MONSTERGAUSS, University of Toronto, 2002, http://www.noc.utoronto.ca/ mikep/MG/.

(53) Brion, C. E.; Young, J. B.; Litvinyuk, I. V.; Cooper, G. Chem. Phys. 2001, 269, 101.

(54) Ramirez-Solis, A.; Hernandez-Lamoneda, R. Revista Mexicana de Fisica 1996, 42, 911 (see in particular page 919).

(55) McCarthy, I. E.; Weigold, E. Rep. Prog. Phys. 1991, 54, 789. 
(56) Brion, C. E. Int. J. Quant. Chem. 1986, 29, 1397.

(57) Lohmann, B.; Weigold, E. Phys. Lett. 1981, 86A, 139.

(58) Mulliken, R. S. J. Chem. Phys. 1935, 3, 517.

(59) Mulliken, R. S. J. Chem. Phys. 1935, 3, 506.

(60) Hood, S. T.; Weigold, E.; McCarthy, I. E.; Teubner, P. J. O. Nature Physical Science 1973, $243,65$.

(61) Hood, S. T.; Hamnett, A.; Brion, C. E. Chem. Phys. Letters 1976, 39, 252.

(62) Hood, S. T.; Hamnett, A.; Brion, C. E. J. Electron Spectrosc. 1977, 11, 205.

(63) Liu, Y.; Cheung, L. F.; Ning, C. G. Chin. Phys. B 2014, 23, 063403-1.

(64) Martin, R. B. J. Chem. Educ. 1988, 65, 668.

(65) Laing, M. J. Chem. Educ. 1987, 64, 124.

(66) Stowasser, R.; Hoffmann, R. J. Amer. Chem. Soc. 1999, 121, 3414.

(67) Baerends, E. J.; Gritsenko, O. V. J. Phys. Chem. A 1997, 101, 5383.

(68) Simoes, A. "The Scientist as Educator and Public Citizen: Linus Pauling and His Era." Conference Oregon State University, October 29 - 30, 2007, Video: “Textbooks as Manifestos: C. A. Coulson after Linus Pauling and R. Mulliken”, Oregon State University Special Collections and Archives, Interview of Ana Simoes by Mary Jo Nye.

(69) Simoes, A.; Gavroglu, K. in Early Ideas in the History of Quantum Chemistry, Chemical Sciences in the 20th Century: Bridging Boundaries, edited by C. Reinhard (Wiley-VCH, Weinheim, 2001).

(70) Pauling, L. The Nature of the Chemical Bond and the Structure of Molecules and Crystals (Third edition, Cornell University Press, New York, 1960).

(71) Penotti, F.; Gerratt, J.; Cooper, D. L.; Raimondi, M. J. Mol. Struct. (Theochem.) 1988, $169,421$.

(72) Ogilvie, J. J. Chem. Educ. 1990, 67, 280.

(73) Scerri, E. R. J. Chem. Educ. 2000, 77, 1492. 
(74) Leung, K. T.; Brion, C. E. J. Amer. Chem. Soc. 1984, 106, 5859.

(75) Leung, K. T.; Brion, C.E. Chem. Phys. 1983, 82, 113.

(76) Litvinyuk, I. V.; Young, J. B.; Zheng, Y.; Brion, C. E. Chem. Phys. 2001, 263, 195.

(77) Zheng, Y.; Neville, J. J.; Brion, C. E. Science 1995, 270, 786.

(78) Neville, J. J.; Zheng, Y.; Brion, C. E. J. Amer. Chem. Soc. 1996, 118, 10533.

(79) Pascual, J. I.; Gomez-Herrero, J.; Rogero, C.; Baro, A. M.; Sanchez-Portal, D.; Artacho, E.; Ordejon, P.; Soler, J. M. Phys. Lett. 2000, 321, 78.

(80) Itani, J.; Levesque, J.; Zeidler, D.; Niikura, H.; Pepin, H.; Kieffer, J-C.; Corkum, P.; Villeneuve, D. M. Nature (London) 2004, 423, 867.

(81) Puschnig, P.; Berebile, S.; Fleming, A. J.; Emtsev, G. K.; Seyller, T.; Riley, J. D.; Ambrosch-Draxi, C.; Netzer, F. P.; Ramsey, M. G. Science 2009, 326, 702.

(82) Luftner, D.; Ules, T.; Reinisch, E. M.; Koller, G.; Soubatch, S.; Tautz, F. S.;

Ramsey, M. G.; Puschnig, P. Proc. Nat. Acad. Sci. 2014, 111, 605.

(83) Cardellini, L. J. Chem. Educ. 2007, 84, 1631.

(84) Grushow, A. J. Chem. Educ. 2011, 88, 860.

(85) McMurry, J. Organic Chemistry (Brooks-Cole, New York, 2000).

(86) Jones Jr., M. Organic Chemistry (W.W. Norton and Co., New York, 1997).

(87) Bruice, P. Y. Organic Chemistry, (Prentice-Hall, New Jersey, 1998).

(88) Solomons, T. W. G. Organic Chemistry (John-Wiley, New York, 1996).

(89) Gillespie, R.J. J. Chem. Educ. 1970, 47, 18.

(90) Weisberg, M. Philosophy of Science 2008, 75, 932. 


\section{Author Information Note}

Present addresses:

C.E. Brion, brion@chem.ubc.ca, P.O. Box 137, Hope, BC V0X 1L0, Canada.

Z. Shi, Energy, Mining and Environment Portfolio, National Research Council Canada, 4150

Wesbrook Mall, Vancouver, BC V6T 1W5, Canada.

G. Cooper, Department of Chemistry, McMaster University, 1280 Main St. W., Hamilton, ON, L8S 4M1, Canada.

Y. J. Zheng: 2772-1055 West Georgia Street, Vancouver, BC V6Z 3P3 Canada. 


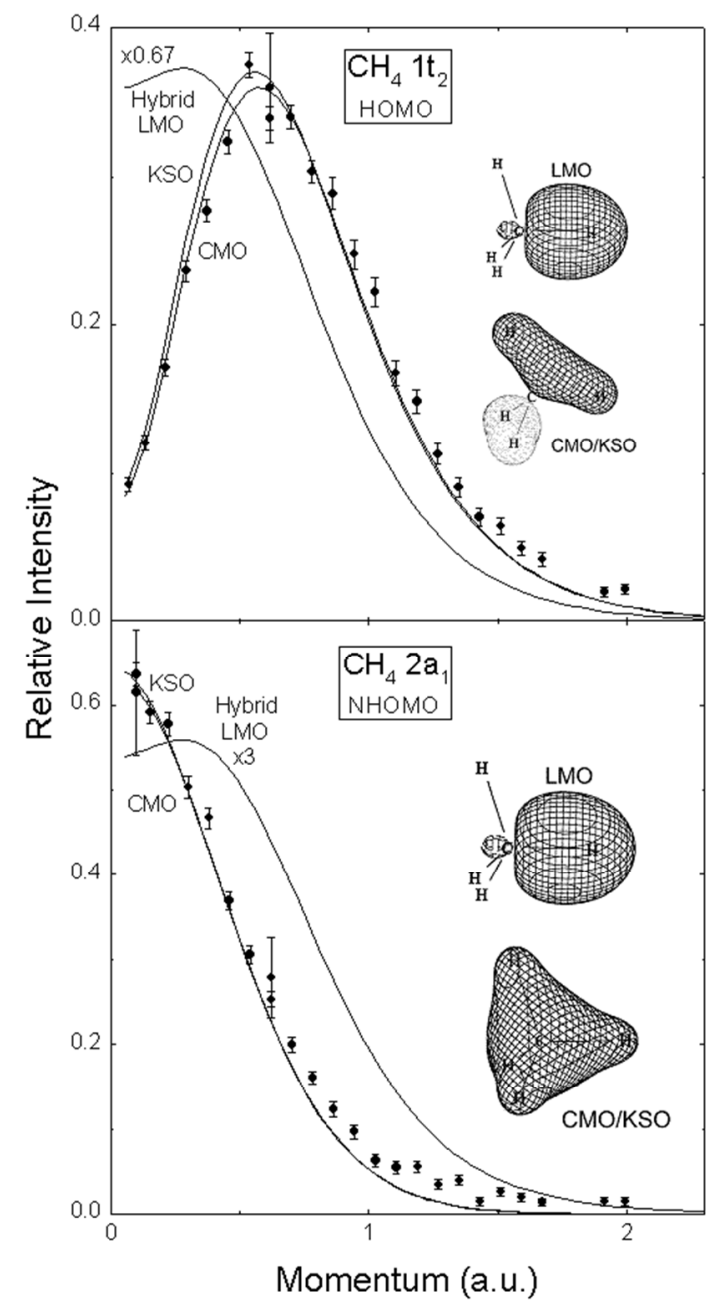

FIG. 1. Calculations and EMS measurements of the electron momentum distributions for the valence electrons of $\mathrm{CH}_{4}$ - see text for further details 


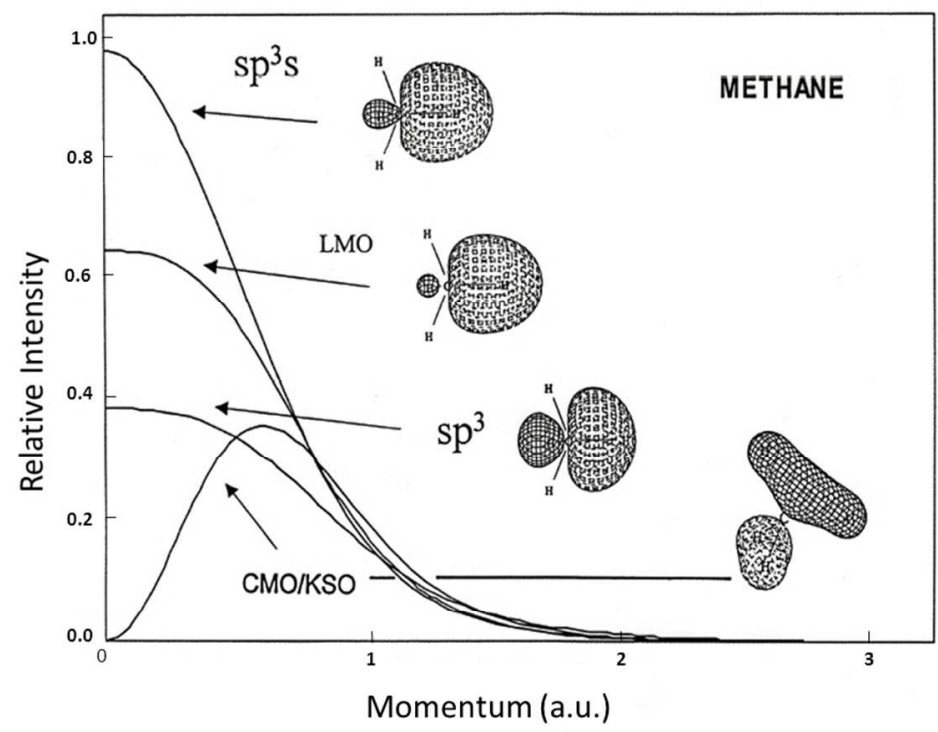

FIG. 2. Calculated electron momentum distributions for methane using localized and delocalized orbital models - see text for further details. 


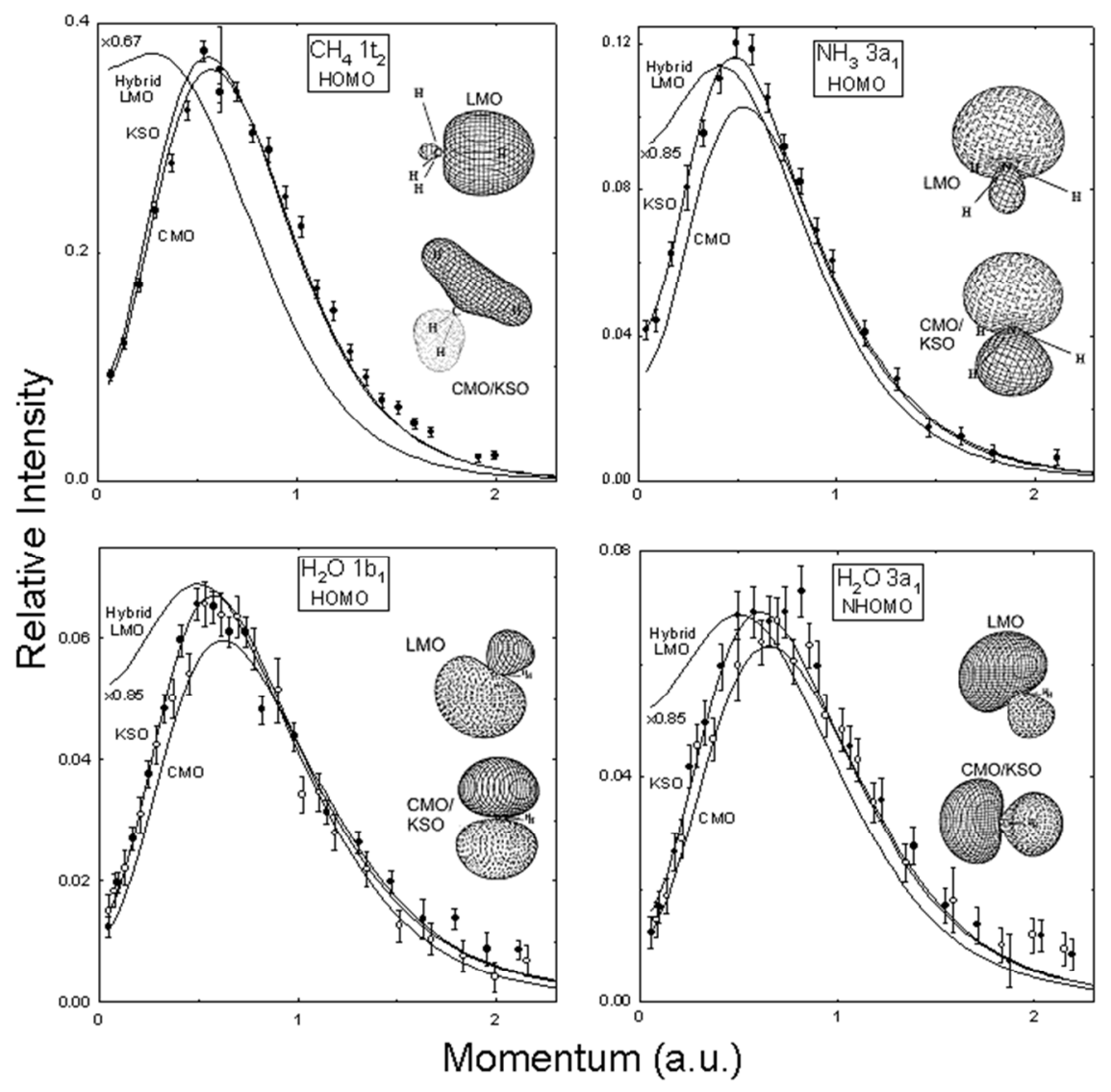

FIG. 3. Calculations and EMS measurements of the electron momentum distributions for the outer valence electrons of $\mathrm{CH}_{4}, \mathrm{NH}_{3}$ and $\mathrm{H}_{2} \mathrm{O}$ - see text for further details. 